Bull. Fac. Agric., Cairo Univ.,65: 10-20 (2014).

\title{
A STUDY ON THE PLANT DIVERSITY IN WADI HAGUL, EASTREN DESERT, EGYPT
}

(Received: 13. 3. 2014)

\author{
By \\ A. A. Mohamed \\ Flora and Phytotaxonomy Research Department, Horticultural Research \\ Institute, Agricultural Research Center, Dokki, Egypt
}

\begin{abstract}
The present study on the plant diversity in Wadi Hagul comprises 82 species belonging to 71 genera from one Gymnospermae family and 25 Angiospermae families: Compositae is represented by 20 species, Leguminosae 9 species, Zygophyllaceae 7 species, and both of Chenopodiaceae and Gramineae are represented by 6 species. Asclepiadaceae and Polygonaceae are represented by 4 species each. Three species from Cruciferae are recorded. Each of Boraginaceae, Resedaceae, Scrophulariaceae, Solanaceae and Umbelliferae are represented by 2 species, while one species is recorded from each of Capparaceae, Cucurbitaceae, Ephedraceae, Euphorbiaceae, Geraniaceae, Labiatae, Malvaceae, Neuradaceae, Nitrariaceae, Plantaginaceae, Ranunculaceae, Tamaricaceae and Thymelaeaceae. The annual species in the studied area are 33 species; while the perennials are 28 species. Nineteen species are recorded as shrubs while the trees represent 2 species.
\end{abstract}

Key words: Eastern desert, Egypt, plant diversity, wadi Hagul

\section{INTRODUCTION}

Wadi Hagul is located in the northern part of the Eastern Desert of Egypt within Cairo-Suez district, delimited by latitudes $29^{\circ} 48^{\prime} 28^{\prime \prime}-$ $29^{\circ} 57^{\prime} 43^{\prime \prime} \quad$ N., and longitudes $32^{\circ} 09^{\prime} 32^{\prime \prime}-$ $32^{\circ} 17^{\prime} 27^{\prime \prime}$ E. Wadi Hagul area is considered a morphotectonic depression falling between the southern scarps of Gebel Ataqa in the north and the northern scarp of El-Galala El-Bahariya Plateau in the south. Generally, the elevation rises gradually westwards until it merges into the great limestone plateau of Eocene age forming the center of the northern part of Eastern Desert. Several works were conducted on the Egyptian deserts and wadies. Abdallah et al. (1972) recorded one species of mosses and 307 species representing 194 genera and 54 families of phanerogames in Nubia, whereas Belal and Springuel (1996) recorded 127 species from Wadi Allaqi. A total of 110 species belonging to 35 families, were recorded in Wadi Allaqi (Ali et al., 1997). The study of natural vegetation on El-Qantara area, North Sinai revealed 113 species from 45 families (Amer 2004). Mosallam (2007) recorded 124 species belonging to 108 genera and 42 families in Saint Katherine protectorate; while Soliman (2007) recorded 50 species in a survey of five wadies (Arbeien, Ithla, Sheraji, Talaa and
Tinya) in south Sinai. El Hadidi (1993) reviewed briefly the main features of the natural vegetation and the different habitat types in the Eastern Deserts. Hassan (1987) conducted a study for the flora of the area extending between Cairo-Suez road in the north and IdfuMersa Alam road in the south. His studies showed apparent diversity in the life forms ranging between woody perennial desert plants to herbaceous submerged water plants. A total of 433 species including 9 endemic were recorded from the study area. The present study aims to: 1- Study the plant diversity of the flora of the desert land in Wadi Hagul 2Documentation the recorded species by herbarium specimens.

\section{MATERIAL AND METHODS}

The study was conducted in Wadi Hagul during the period from 2011 to 2013. Eight visits were performed to the area during this period. Seven sites (S1-S7) were selected to cover the species diversity in the area (Fig. 1). About 550 specimens were collected and prepared as herbarium sheets. These herbarium sheets were preserved in the herbarium of the Flora and Phytotaxonomy Researches Department (CIAM) and arranged according to Engler's system. The specimens were identified 


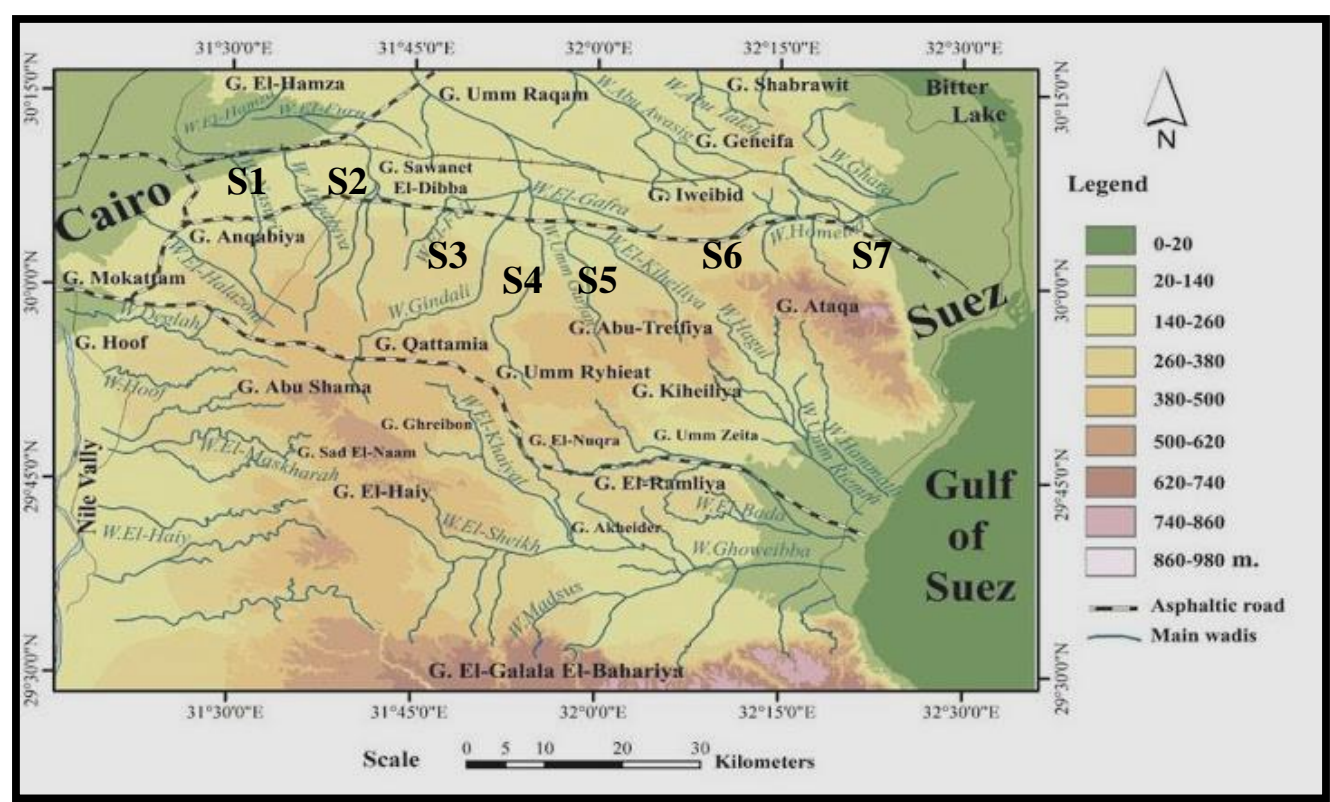

Fig. (1): A map showing the studied area in Wadi Hagul.

according to Täckholm (1974), Boulos, (1999, 2000, 2002 and 2005). The species recorded were arranged alphabetically within their families. For each species original publication, reference, habitat and life form, floristic category and local names were cited. A map for the studied area is shown Fig. (1).

\section{RESULTS}

The following are a detailed list of the 82 species mentioned in Table 1 together with their habitats, life forms, floristic categories and local names.

For floristic categories the following abbreviations are used: IR -Tur = Irano -

Table (1): Species recorded from Wadi Hagul with its families and habits according to Boulos (1999-2005).

\begin{tabular}{|c|c|c|}
\hline Taxa & Family & Habitat \\
\hline Acacia tortilis (Forssk.) Hayne subsp. raddiana (Savi) Brenan & Leguminosae & Tree \\
\hline Achillea fragrantissima (Forssk.) Sch. Bip. & Compositae & Perennial \\
\hline Alhagi graecorum Boiss. & Leguminosae & Perennial \\
\hline Anabasis articulata (Forssk.) Moq. & Chenopodiaceae & Shrub \\
\hline Anabasis setifera Moq. & Chenopodiaceae & Shrub \\
\hline Asteriscus hierochuntius (Michon) Wiklund & Compositae & Annual \\
\hline Astragalus vogelii (Webb) Bornm. & Leguminosae & Annual \\
\hline Bassia muricata (L.) Asch. & Chenopodiaceae & Annual \\
\hline Brassica tournefortii Gouan & Curciferae & Annual \\
\hline Calotropis procera (Aiton) W. T. Aiton & Asclepiadaceae & Shrub \\
\hline $\begin{array}{l}\text { Calligonum polygonoides L. subsp. comosum } \\
\text { ( L' Her. ) Soskov }\end{array}$ & Polygonaceae & Shrub \\
\hline Centaurea aegyptiaca $\mathrm{L}$ & Compositae & Annual \\
\hline Chenopodium murale L. & Chenopodiaceae & Annual \\
\hline Citrullus colocynthis (L.) Schrad. & Cucurbitaceae & Perennial \\
\hline Cleome droserifolia (Forssk.) Delile & Capparaceae & Shrub \\
\hline Cotula cinerea Delile & Compositae & Annual \\
\hline Cynanchum acutum L. subsp. acutum & Asclepiadaceae & Perennial \\
\hline Deverra tortuosa (Desf.) DC & Umbelleferae & Shrub \\
\hline Diplotaxis harra (Forssk.) Boiss. & Cruciferae & Perennial \\
\hline Echinops spinosus L. & Compositae & Perennial \\
\hline Emex spinosa (L.) Campd. & Polygonaceae & Annual \\
\hline Ephedra alata Decne. & Ephedraceae & Shrub \\
\hline $\begin{array}{l}\text { Erodium laciniatum (Cav.) Willd. subsp. pulverulentum (Boiss.) } \\
\text { Batt. in Batt. \& Trab. }\end{array}$ & Geraniaceae & Annual \\
\hline Euphorbia retusa Forssk. & Euphorbaiaceae & Perennial \\
\hline Fagonia arabica $L$. & Zygophyllaceae & Perennial \\
\hline
\end{tabular}


Table (1): Cont.

\begin{tabular}{|c|c|c|}
\hline Fagonia bruguieri DC & Zygophyllaceae & Perennial \\
\hline Fagonia mollis Delile & Zygophyllaceae & Perennial \\
\hline Filago desertorum Pomel & Compositae & Annual \\
\hline Foeniculum vulgare Mill. & Umbelliferae & Perennial \\
\hline Haloxylon salicornicum (Moq.) Bunge ex Boiss. & Chenopodiaceae & Shrub \\
\hline Heliotropium arbainense Fresen. & Boraginaceae & Perennial \\
\hline Hordeum murinum L., subsp. leporinum (Link) Arcang. & Gramineae & Annual \\
\hline Hyoscyamus muticus $\mathrm{L}$. & Solanaceae & Perennial \\
\hline Ifloga spicata (Forssk.) Sch. Bip. & Compositae & Annual \\
\hline Imperata cylindrica $($ L.) Raeusch & Gramineae & Perennial \\
\hline Iphiona mucronata (Forssk.)Ash. \& Schweinf. & Compositae & Perennial \\
\hline Kickxia aegyptiaca (L.) Nabelek subsp. aegyptiaca & Scrophulariaceae & Perennial \\
\hline Launaea capitata (Spreng.) Dandy in F. W. Andrews & Compositae & Perennial \\
\hline $\begin{array}{l}\text { Launaea mucronata (Forssk.) Muschl. subsp. cassiniana (Jaub. } \\
\& \text { Spach) N. Kilian }\end{array}$ & Compositae & Annual \\
\hline Launaea nudicaulis (L.) Hook. f. & Compositae & Perennial \\
\hline Launaea spinosa (Forssk.) Sch. Bip. ex Kuntze & Compositae & Perennial \\
\hline Lavandula coronopifolia Poir. in Lam. & Labiatae & Perennial \\
\hline Leptadenia pyrotechnica (Forssk.) Decne. & Asclepiadaceae & Shrub \\
\hline Lotus glaber Mill. & Leguminosae & Perennial \\
\hline Lycopersicum esculentum Miller & Solanaceae & Annual \\
\hline Malva parviflora $\mathrm{L}$. & Malvaceae & Annual \\
\hline Medicago lupulina $\mathrm{L}$. & Leguminosae & Annual \\
\hline Medicago polymorpha $\mathrm{L}$. & Leguminosae & Annual \\
\hline Nauplius graveolens (Forssk.) Wiklund & Compositae & Shrub \\
\hline Neurada procumbens $\mathrm{L}$. & Neuradaceae & Annual \\
\hline Nigella sativa $\mathrm{L}$. & Ranunculaceae & Annual \\
\hline Nitraria retusa (Forssk.) Asch. & Nitrariaceae & Shrub \\
\hline Ochradenus baccatus Delile & Resedaceae & Shrub \\
\hline Pennisetum divisum (Forssk ex J. F. Gmel.) Henrard & Gramineae & Perennial \\
\hline Pergulaia tomentosa $\mathrm{L}$. & Asclepiadaceae & Perennial \\
\hline Phragmites australis (Cav.) Trin. ex Steud. & Gramineae & Perennial \\
\hline Plantago ovata Forssk. & Plantaginaceae & Annual \\
\hline Polygonum equisetiforme $\mathrm{Sm}$. & Polygonaceae & Perennial \\
\hline Pulicaria undulata (L.) C.A.Mey. & Compositae & Shrub \\
\hline Reichardia tingitana (L.) Roth & Compositae & Annual \\
\hline Reseda pruinosa Delile & Resedaceae & Annual \\
\hline Retama raetam (Forssk.) Webb \& Berthel. & Leguminosae & Shrub \\
\hline Rumex vesicarius $\mathrm{L}$. & Polygonaceae & Annual \\
\hline Salsola imbricata Forssk. & Chenopodiaceae & Perennial \\
\hline Schismus barbatus (L.) Thell. & Gramineae & Annual \\
\hline Scrophularia xanthoglossa Boiss. & Scrophulariaceae & Perennial \\
\hline Senecio glaucus L. subsp. coronopifolius (Maire) C. Alexander & Compositae & Annual \\
\hline Seriphidium herba-album (Asso) Soják & Compositae & Shrub \\
\hline Sonchus oleraceus $L$. & Compositae & Annual \\
\hline Stipa canpensis Thunb. & Gramineae & Annual \\
\hline Symphyotrichum squamatum (Spreng.) Nesom & Compositae & Annual \\
\hline Tamarix nilotica (Ehrenb.) Bunge & Tamaricaceae & Tree \\
\hline Thymelaea hirsuta (L.) Endl. & Thymelaeaceae & Shrub \\
\hline Tribulus terrestris $L$. & Zygophyllaceae & Annual \\
\hline Trichodesma africanum (L.) R. Br. & Boraginaceae & Perennial \\
\hline Trigonella glabra Thunb. & Leguminosae & Annual \\
\hline Trigonella stellata Forssk. & Leguminosae & Annual \\
\hline Volutaria lippii (L.) Cass. ex Maire & Compositae & Annual \\
\hline Zilla spinosa (L.) Prantl & Cruciferae & Shrub \\
\hline Zygophyllum coccineum $\mathrm{L}$. & Zygophyllaceae & Shrub \\
\hline Zygophyllum decumbens Delile & Zygophyllaceae & Shrub \\
\hline Zygophyllum simplex $\mathrm{L}$. & Zygophyllaceae & Annual \\
\hline
\end{tabular}


Turanian; Euro $-\mathrm{Sib}=$ Euro - Siberian; Med $=$ Mediterranean; Sah - Sind = Saharo-Sindian; Sud - Zamb $=$ Sudano - Zambezian .

* Refer to the species recorded with a new distribution according to Täckholm 1974 and Boulos 2009.

\section{Asclepiadaceae}

1.1. Calotropis procera (Aiton) W. T. Aiton, Horb. Kew., ed. 1,1: 305 (1789); Boulos 2000, p. 220.

Habitat and life form: desertic plant. Nanophanerophyte.

Floristic category: Sah - Sind, IR - Tur and Sud - Zamb Regions.

Local names: 'Oshar, 'Oshaar, 'Oshr, 'Osher, Abat, Abbad, Beid el - 'Oshar ( fruit ).

2.2. Cynanchum acutum L., Sp. Pl., ed. 1, 212 (1753); subsp. acutum, Boulos 2000, p.222

Habitat and life form: Waste moist ground, Nile and canal banks. Therophyte.

Floristic category: Med, IR-Tur and Sah-Sind Regions.

Local names: 'Ollaiq, 'Olleiq, Modeid, Motteit, Libbein, Libbeina.

3.3. Leptadenia pyrotechnica (Forssk.) Decne., Ann. Sci. Nat. Bot., sêr. 2, 9: 270 (1838); Boulos 2000, p. 225.

Habitat and life form: Sandy desert plains and wadis. Nanophanerophyte.

Floristic category: Sah-Sind, IR-Tur and SudZamb Regions.

Local names: Markh, Agwaam, Seisabaan, Sesebaan.

4.4. Pergulaia tomentosa L., Syst. Nat., ed. 12, 2: 191 (1767); Boulos 2000, p. 223.

Habitat and life form: desertic plant. Chamaephyte.

Floristic category: Sah-Sind and IR-Tur Regions.

Local names: Ghalqa, Gahlqay, Atma, Laban el-Homara, Omm. El-Laban, Dimish el- ghalq.

\section{Boraginaceae}

5.5. Heliotropium arbainense Fresen., Mus. Senckenb. 1: 168 (1834); Boulos 2000, p.276.

Habitat and life form: Sandy and stony desert wadis and plains, edges of cultivated ground.. Chamaephyte.

Floristic category: Sah - Sind and Sud - Zamb Regions.

Local names: Dafaara, Erhaba, 'Atana.
6.6. Trichodesma africanum (L.) R. Br., Prodr. Fl. Nov. Holl. 1: 496 (1810); Boulos 2000, p. 286.

Habitat and life form: Sandy and gravelly wadis, sandy desert plains, edges of cultivation. Chamaephyte.

Floristic category: Sah - Sind Sud-Zamb and IR - Tur Regions.

Local names: Hameem, Lisaan et-teir, Himhim, Horraaqa, Horreiq Shoak ed-dab'a, Khodaar, Tadatt, Shana, Losseiq, Lesseq, Lisseq, Lossaaq, Lessaaq.

\section{Capparaceae}

7.7. Cleome droserifolia (Forssk.) Delile, Descr. Egypte, Hist. Nat. 250 (1814); Boulos 1999, p. 177.

Habitat and life form: Stony and sandy wadis and plains. Chamaephyte.

Floristic category: Sah-Sind Region.

Local names: 'Afein, 'Afen, Mashta, Mishtar, Reeh el-bard.

\section{Chenopodiaceae}

8.8. Anabasis articulata (Forssk.) Moq., in A. DC., Pordr. 13(2): 212 (1849); Boulos 1999, p. 126.

Habitat and life form: Stony and sandy wadis. Chamaephyte.

Floristic category: Med and Sah-Sind Regions. Local names: Tarteer, Balbal, Belbal, 'Ageram, Hamd.

8.9. Anabasis setifera Moq., Chenop. Monogr. Enum. 164 (1840); Boulos 1999, p. 125.

Habitat and life form: Stony and sandy wadis. Chamaephyte.

Floristic category: Med and Sah-Sind Regions. Local names: Shabb, 'Agram, Hamd, Djell, Djill, Assal.

9.10. Bassia muricata (L.) Asch. in Schweinf., Beiter. Fl., Aethiop.: 187 (1867); Boulos 1999, p. 106.

Habitat and life form: Weed in new reclaimed lands with sandy soils. Therophyte.

Floristic category: Sah - Sind and IR - Tur Regions.

Local names: Ghobbeira, Ghobbayra, Layya, Haythaam.

10.11. Chenopodium murale L., Sp. Pl., ed. 1, 219 (1753); Boulos 1999, p. 96.

Habitat and life from: Weed. Therophyte.

Floristic category: Cosmopolitan.

Local names: Lissan el-teir ( el-thor ), Qehaniya, Qeihaniya, Abu 'efin ( 'efeina), Fiss el- kelb(kilaab) Sontaar, Fatteh, Shagaret elmotteina, Fisseih, Fosseish, Sakaraan, Sekraan. 
11.12. Haloxylon salicornicum (Moq.) Bunge ex Boiss., Fl. Orient. 4: 949 (1879); Boulos 1999, p. 124.

Habitat and life form: Sandy and stony deserts and wadis. Nanophanerophyte.

Floristic category: Sah - Sind and IR - Tur Regions.

Local names: Rimth, Remeh, Balbal, Belbel..

12.13. Salsola imbricata Forssk., Fl. Aegypt.Arab. CVII, CVIII. 57 (1775); Boulos1999, p. 118.

Habitat and life form: desertic plants. Chamaephyte.

Floristic category: Sah - Sind and IR - Tur Regions.

Local names: Tarteer, Khreysy, Khreit, Haad, Shinaan, Molleih, Molleyh, Mellikh.

\section{Compositae}

13.14. Achillea fragrantissima (Forssk.) Sch. Bip., Flora (Regensburg) 38: 13 (1855); Boulos 2002, p. 249.

Habitat and life form: Desert wadis and sandy plains. Nanophanerophyte.

Floristic category: Sah-Sind Region.

Local names: Gesoom, Gesoom gebeli, Alegiaan, Eileiaan, Baboonig, Baboonag, Qaysoom, Qasoom, Qesoom, Be'eithraan,

Ba'ataraan.

14.15. Asteriscus hierochuntius (Michon) Wiklund, Nord. J. Bot. 5: 307 (1985); Boulos 2002, p. 225.

Habitat and life form: Sandy andstony desert wadis and plains. Therophyte.

Floristic category: Sah-Sind Region.

Local names: Unknown.

15.16. Centaurea aegyptiaca L., Mant. 118 (1767); Boulos 2002, p. 168.

Habitat and life form: Desert wadis. Therophyte.

Floristic category: Sah-Sind Region.

Local names: Moraar, Shoak 'antar, Yamroor, Yamraar, Netesh.

16.17. Cotula cinerea Delile, Descr. Egypte, Hist. Nat. 275,t. 47, f.4 (1814); Boulos 2002, p. 257.

Habitat and life from: Winter weed. Therophyte.

Floristic category: Cosmopolitan.

Local names: Ribyaan, Erbeyaan, Arbeyanny, Sakaraan, Sekraan.

17.18. Echinops spinosus L., Mant. 119. (1767); Boulos 2002, p. 146.

Habitat and life form: desertic plant, Chamaephyte.

Floristic category: Med Region.
Local names: Khasheer, also Khharshoof, Khosheroof, Khoshroof, Khashroof (names for all thistles ).

18.19. Filago desertorum Pomel, Nouv. Mat. Fl. Atlant. 1: 46 (1874); Boulos 2002, p. 199.

Habitat and life form: Desert wadis and plains.

Therophyte.

Floristic category: Med, Sah-Sind and Ir-Tur Regions.

Local names: Kurbat.

19.20. Ifloga spicata (Forssk.) Sch. Bip. in Webb \& Berthel., Phyt. Canar. 2: 310 (1845); Boulos 2002, p. 197.

Habitat and life form: Sandy wadis and desert plains. Therophyte.

Floristic category: Med and Sah-Sind Regions.

Local names: Kreishit el-gadye, Shagaret elma'eeza, Kareish shedi, Kheishit es-sanheela, Gomborr.

20.21. Iphiona mucronata (Forssk.) Ash. \& Schweinf., Mém. Inst. Egypt. 2:86 (1887); Boulos 2002, p. 244.

Habitat and life form: desertic plant. Chamaephyte.

Floristic category: Sah - Sind Region.

Local names: Dafeera, Dhafara, Dhafra, Dhofra, Dafra, Dafri.

21.22. Launaea capitata (Spreng.) Dandy in F. W. Andrews, Fl. Pl. Sudan 3: 40 (1956); Boulos 2002, p. 294.

Habitat and life from: Desert wadis, Sandy and alluvial plains, edges of cultivation, often a weed in reclaimed desert land. Chamaephyte.

Floristic category: Sah - Sind, Med and SudZamb Regions.

Local names: 'Adeed, Hoadaan, Halawet elghozlaan, Hawthaan, El-anayaat, Shagaret ellibbeina, Howei kelaab, Halawaan.

21.23. Launaea mucronata (Forssk.) Muschl., Man. Fl. Egypt 2: 1057 (1912) subsp. cassiniana (Jaub. \& Spach) N. Kilian, Willdenowia 25: 277 (1995); Boulos 2002, p. 298.

Habitat and life form: Sandy and stony deserts, alluvial plains, edges of cultivation. Therophyte.

Floristic category: Sah-Sind and Sud-Zamb Regions.

Local names: Amroor, Maroor, Yamroor, Morreira-entiya, 'Addeid, Haddieida.

21.24. Launaea nudicaulis (L.) Hook. f., Fl. Brit. Ind. 3:416 (1881); Boulos 2002, p. 291.

Habitat and life from: Sandy and alluvial soils, 
desert plains and wadis, edges of cultivation. Chamaephyte.

Floristic category: Sah - Sind, Med and IR Tur Regions.

Local names: Ribaay eilaab, Howa, Hawa, Howei, Moraar, Howthaan, Lesseq, Lesseiq, Lisseq, Lossaaq, Lessaaq.

21.25. Launaea spinosa (Forssk.) Sch. Bip. ex Kuntze, Revis. Gen. PI. 1: 350 (1891); Boulos 2002, p. 291.

Habitat and life form: Rocky deserts and escarpments. Chamaephyte.

Floristic category: Sah-Sind Region.

Local names: Kebaath, Kabath, Keddaad, Zaggwa.

22.26. Nauplius graveolens (Forssk.) Wiklund, Nord. J. Bot. 7: 16 (1987); Boulos 2002, p. 226.

Habitat and life form: Desert wadis and dry lands. Chamaephyte.

Floristic category: Sah-Sind Region.

Local names: Rabd, Rabl, Rabool, Beheyma, Noqd, Noqod, Moqqeid.

23.27. Pulicaria undulata (L.) C.A.Mey, Verz. Pfl. Casp. Meer. 79 (1831);

Boulos 2002, p. 221.

Habitat and life form: desertic plant. Chamaephyte.

Floristic category: Sud - Zamb and Sah - Sind Regions.

Local names: Kootkaat, Rabool, Rabl, Rabd, Ghobbayra, Shaay gabali.

24.28. Reichardia tingitana (L.) Roth, Bot. Abh. 35 (1787); Boulos 2002, p. 299.

Habitat and life form: Desert wadis, sandy and alluvial plains, edges of cultivation. Therophyte.

Floristic category: Med and Sah-Sind Regions. Local names: Morrar, Hawwa.

25.29. Senecio glaucus L., Sp. Pl., ed. 1, 868 (1753) subsp. coronopifolius (Maire) C. Alexander, Notes Roy. Bot. Gard. Edinb. 37: 412 (1979); Boulos 2002, p. 262.

Habitat and life form: Winter weed especially in new reclaimed lands. Therophyte.

Floristic category: Sah - Sind, Med and IR Tur Regions.

Local names: Moroor, Moreir, Moreyre, Meroor, Sofeera, Safeery, Sofra, Safra,

Sofeir - attan.

Local names: Qorreis, Omm lonein, Omm loweinein.

26.30. Seriphidium herba-album (Asso) Soják, Cas. Nár. Muz. (Prague) 152 (1): 22
(1983); Boulos 2002, p. 261.

Habitat and life form: Sandy and stony deserts and calcareous ridges. Chamaephyte.

Floristic category: Med and Sah-Sind Regions.

Local name: Sheeh.

27.31. Sonchus oleraceus L., Sp. Pl., ed. 1,749

(1753); Boulos 2002, p. 309.

Habitat and life form: Common weed in cultivated lands, along. canal banks and

roadsides. Therophyte.

Floristic and category: Cosmopolitan.

Local names: Go'odied, Goo'dei, Galawein, Galaaweel, Galaeel, Khass, Khaz, Hoa

el- ganayin, Hawa, Howa, Howei, Kass Klab,

Khass wez, Shekhasheikh,

Shikschaakh.

28.32. Symphyotrichum squamatum (Spreng.) Nesom, Phytologia 77:292 (1994), Boulos2002, p. 194.

Habitat and life form: Common weed on Nile and canal banks and in waste places. Therophyte.

Floristic category: Pantropical.

Local names: Losseiq, Lisseq, Lossaq, Lesseq.

29.33. Volutaria lippii (L.) Cass. ex Maire in Jahand. \& Maire, Cat. Pl. Maroc 3: 817 (1934); Boulos 2002, p. 176.

Habitat and life form: Desert wadis and waste ground. Therophyte.

Floristic category: Med and Sah-Sind Regions.

Local names: Moraar, Moreir.

\section{Cruciferae}

30.34.Brassica tournefortii Gouan., III. Observ. Bot. 44, t. ZOA (1773); Boulos 1999, p. 211.

Habitat and life form: Weed in winter crops. especially new reclaimed lands. Therophyte.

Floristic category: Med Region with extension to IR-Tur Region.

Local names: Shiltaam, Shirtaam.

31.35. Diplotaxis harra (Forssk.) Boiss., Fl. Orient. 1: 388 (1867). subsp. harra; Boulos 1999, p. 209.

Habitat and life form: Sandy plains and stony wadis. Chamaephyte.

Floristic category: Sah-Sind and Sud-Zamb Regions.

Local name: Harra.

32.36. Zilla spinosa (L.) Prantl, In Engl. \& Prantl, Natürl. Pflanzemfam. III (2): 175 (1891); Boulos 1999, p. 218.

Habitat and life form: Sandy plains. Chamaephyte.

Floristic category: Sah - Sind Region. 
Local names: Zilla, Silla, Sillet, Besilla, Basilla, Shagaret el- hommoos, Ommo, Hammoos.

\section{Cucurbitaceae}

33.37. Citrullus colocynthis (L.) Schrad., Linnaea 12: 414 (1838); Boulos 2000, p. 140.

Habitat and life form: Sandy plains, desert wadis, edges of cultivation in lands. Chamaephyte.

Floristic category: Sah-Sind and Sud-Zamb Regions.

Local names: Handal, Hanzal, Oorky, Tattoor.

\section{Ephedraceae}

34.38. Ephedra alata Decne., Ann. Sci. Nat. Bot., sér. 2, 2: 239 (1834); Boulos 1999, p. 11.

Habitat and life form: Desert sandy plains. Nanophanerophyte.

Floristic category: Sah-Sind Region.

Local names: 'Alde el-dabbagh, 'Alde elgamal.

\section{Euphorbiaceae}

35.39. Euphorbia retusa Forssk., Fl. Aegypt.Arab. 93 (1775); Boulos 2000, p. 59.

Habitat and life form: Sandy plains and desert wadis. Chamaephyte.

Floristic category: Sah - Sind and Sud-Zamb Regions.

Local names: No'maniya, Melbeyn.

\section{Geraniaceae}

36.40. Erodium laciniatum (Cav.) Willd., Sp. Pl., ed. 4, 3: 633 (1800) subsp. Pulverulentum (Boiss.) Batt. In Batt. \& Trab., Fl. Algerie 1: 126 (1888); Boulos 2000, p. 7.

Habitat and life form: Desert wadis, sandy and gravelly soils. Therophyte.

Floristic and category: Med and Sah-Sind Regions.

Local names: Abu mosfaah, Dahimiya, Dahmiyet ghozlaan, Abu 'oqeila, Bakhtery.

\section{Gramineae}

37.41. Hordeum murinum L., Sp. Pl., ed. 1, 85 (1753) subsp. leporinum (Link) Arcang., Comp. Fl. Ital. 805 (1882); Boulos 2005, p. 207.

Habitat and life form: Weed in fields and moist ground. Therophyte.

Floristic category: Med and IR-Tur Regions.

Local names: Sheer Barri, Sheera.

38.42. Imperata cylindrica (L.) Raeusch., Nom. Bot., ed. 3: 10 (1797); Boulos 2005, p. 327.

Habitat and life form: Canal banks, roadsides and waste ground. Chamaephyte.
Floristic category: Pantropical.

Local names: Halfa, Deil el-qott, Silla, Sill, Bodweya, Beniesh-shaam, Ambarta.

39.43. Pennisetum divisum (Forssk ex J. F. Gmel.) Henrard, Blumea 3: 162 (1938); Boulos 2005, p. 314.

Habitat and life form: Sandy deserts. Chamaephyte.

Floristic category: Sah-Sind Region.

Local names: Thommaam, Themmaam, Dah, Ratam, Retam, Retem, Morraat-ghazal, Karaay, Theram, Hada.

40.44. Phragmites australis (Cav.) Trin. ex Steud., Nomencl. Bot., ed. 2, 2: 324 (1841); Boulos 2005, p. 223.

Habitat and life form: Moist places and banks of the Nile and irrigation canals.

Helophyte.

Floristic category: Pantropical.

Local names: Hagna, Hagana, Ghaab, Ghaab reehi Boos, Boos khabba

Boos baw, Qasaba, Qasab, Qassaba, Qasab hegaazi, Qesba, Hoshanaat, Heesh,

Heesh- maddaad, Taghaaneenet, Tighaaminee'n, Taneimme, Aghaaneem, Ighoonaam, Moddeid, Meddaad, Mottiet.

41.45. Schismus barbatus (L.) Thell., Bull. Herb. Biossier, se'r. 2, 7: 391 (1756); Boulos 2005, p. 218.

Habitat and life form: Sandy and gravelly soils. Therophyte.

Floristic category: Euro-Sib, IR-Tur and Med Regions.

Local names: Bahma, Bohma, Abumaashi, Safsoof, Zaghab (Zera') el-faar, Abu hereiba (hareeba), Khafoor.

42.46. Stipa canpensis Thunb., Prodr. Fl. Cap. 1: 19 ( 1794 ); Boulos 2005, p. 132.

Habitat and life form: Desertic plant. Therophyte.

Floristic category: Sah - Sind and Med Regions.

Local names: Sabat, Sabad, Sabt, Safsoof, Sabal (Sebl) abu el- Hossein, Abufakhoor,Tera, Dreira, Dreira.

\section{Labiatae}

43.47. Lavandula coronopifolia Poir. in Lam., Encycl. Suppl. 3: 308 (1813); Boulos 2002, p.11.

Habitat and life form: Calcareous ground, desert wadis. Chamaephyte.

Floristic category: Sah-Sind Sud-Zamb and IrTur Regions.

Local names: Zeita, Zeiti, Balah, Diktae, Natash, 
Netash, Netesh.

13. Leguminosae
44.48. Acacia tortilis (Forssk.) Hayne, Getreue Darstell. Gew. 10, t. 31 (1827). subsp. raddiana (Savi) Brenan, Kew Bull. 12: 87 (1927); Boulos 1999, p. 368.

Habitat and life form: Sandy plains and desert wadis. Phanerophyte.

Floristic category: Sah-Sind and Sud-Zamb Regions.

Local names: Sayaal, Samoor, Saganeib, Towaay, Ollaaf, Samraa, Samrahl.

45.49. Alhagi graecorum Boiss., Diagn. Pl. Orient. 9: 114 (1849); Boulos 1999, p. 240.

Habitat and life form: Moist waste ground and edges of cultivation. Hemi-cryptophyte

Floristic category: Palaeotropical.

Local names: 'Aqool, 'Agool, Shoak, Shoak elgamal, Afisoor.

46.50. Astragalus vogelii (Webb) Bornm., Beih. Bot Centraalbl. 33 (2): 233 (1915); Boulos 1999, p. 329.

Habitat and life form: Sandy and desert wadis. Therophyte.

Floristic category: Sah-Sind and Sud-Zamb Regions.

Local names: Qarn, Qarna, Qarni, Horboss, Taweel, Fag'ai.

47.51. Lotus glaber Mill., Gard. Dict., ed. 8, no. 3 (1768); Boulos 1999, p. 295.

Habitat and life form: Moist and cultivated ground, canal banks, lawns. Chamaephyte.

Floristic category: Med and Sah-Sind Regions.

Local names: Qarn el-ghazaal, Rigl el-asfoor, Zeita, Zeiti.

*48.52. Medicago lupulina L., Sp. Pl., ed. 1, 779 (1753); Boulos 1999, p. 269.

Habitat and life form: Moist ground, edges of cultivation. Therophyte.

Floristic category: Med and Sah-Sind Regions.

Local names: Nafal, Nafala, Nafla, Nefel.

48.53. Medicago polymorpha L., Sp. Pl., ed. 1, 779 (1753); Boulos 1999, p. 269.

Habitat and life form: Cultivated, waste and moist ground. Therophyte.

Floristic category: Cosmopolitan.

Local names: 'Oqqeil, Nafal, Nafala, Nafla, Nefel.

49.54. Retama raetam (Forssk.) Webb \& Berthel., Phyt. Canar. 3 (2, 2): 56 (1842) subsp. raetam; Boulos 1999, p. 258.
Habitat and life form: Desert wadis. Nanophanerophyte.

Floristic category: Sah-Sind Region.

Local names: Ratam, Retam, Retem, Retembehaan.

50.55. Trigonella glabra Thunb. Pl. Cap: 137 (1800); El - Hadidi \& Fayed 1994, p.265.

Habitat and life form: Sandy soils. Therophyte.

Floristic category: Sud - Zamb Med and Sah Sind Regions.

Local names: 'Eshb ( Oshb) el- malik, Daraqraq, Deraaq, Daraaq, Zeraqraaq.

50.56. Trigonella stellata Forssk., Fl. Aegypt.Arab. 140 (1775); Boulos 1999, p. 264.

Habitat and life form: Sandy soils. Therophyte. Floristic category: Med and Sah Sind Regions.

Local names: Gargas, Shetn el-khaadem, Atoor garm, Hesheita, Hisheita.

14. Malvaceae

51.57. Malva parviflora L., Sp. PI., ed. 2,926 (1763); Boulos 2000, p. 94.

Habitat and life form: Winter weed in cultivated lands and along Nile and canal

banks. Therophyte.

Floristic category: Med, Euro - Sib, IR- Tur and Sah - Sind Regions.

Local names: Raqma, Raqmiya, Khobbeiza, Khobaaza.

\section{Neuradaceae}

52.58. Neurada procumbens L., Sp. Pl., ed. 1, 441 (1753); Boulos 1999, p. 249.

Habitat and life form: Sandy plains and desert wadis. Therophyte.

Floristic category: Sah-Sind and Sud-Zamb Regions.

Local names: Losseiq, Lesseq, Lisseq, Lossaaq, Lessaaq, Doreis, Sa'daan, Shangareen.

\section{Nitrariaceae}

53.59. Nitraria retusa (Forssk.) Asch.., Verh. Bot. Vereins. Prov. Brandenb. 18: 94 (1876); Boulos 2000, p. 31.

Habitat and life form: Coastal and inland saline soils, edges of salt marshes. Nanophanerophyte.

Floristic category: Sah-Sind and Ir-Tur Regions.

Local names: Ghardaq, Gharqad, Gherdeq, Sahanoon, 'Agelgaam, 'Adjeldjaam, Timmeir, Tommeir, 'Anab ed-deeb.

\section{Plantaginaceae}

54.60. Plantago ovata Forssk., Fl. Aegypt.Arab. 31 (1775); Boulos 2002, p. 120.

Habitat and life form: Sandy wadis and depressions. Therophyte.

Floristic category: Cosmopolitan. 
Local names: Geneima, Djeneima, Doqeis, Yanam, Yanama, Yenem, Loqmet en-na'ga.

18. Polygonaceae

55.61. Calligonum polygonoides L., Sp. Pl., ed. 1, 333 (1753) subsp comosum ( L' Her. ) Soskov, Nov. Sist.Vyss, Rast .12:153 (1975); Boulos 1999, p.24.

Habitat and life form: desertic plant. Chamaephyte.

Floristic category: Sah - Sind and IR - Tur Regions.

Local names: Arta, Teeb, Risoo, Rosah.

56.62. Emex spinosa (L.) Campd., Monogr. Rumex 58, t. 1 (1819); Boulos 1999, p. 24.

Habitat and life from: Winter weed on cultivations and canal banks. Therophyte.

Floristic category: Med and Sah - Sind Regions with extension to IR - Tur Region.

Local names: Drs el-agooz, Rookbet el-agooz, Shagaret el-o agooz, Batbat, Tablaalast, Keikh, Kolleyly, Gazeesi, Hommeid, Shobeit, Hensaat, Hanzaab, Widna, Figl el-gabl, Losseiq, Lesseq, Lisseq, Lossaaq, Lessaaq.

57.63. Polygonum equisetiforme Sm., Fl. Graec. Prodr. 1: 266 (1809); Boulos 1999, p. 30.

Habitat and life form: Waste ground roadsides. field broders and canal banks.

Chamaephyte.

Floristic category: Med, Sah - Sind and IR Tur Regions.

Local names: Qordaab, Batwa, Okreish, Afraag, Gorthaab.

58.64. Rumex vesicarius L., Sp. Pl.., ed. 1, 336 (1753); Boulos 1999, p. 34.

Habitat and life from: Rocky and sandy desert wadis. Therophyte.

Floristic category: Med, Sah-Sind and IR - Tur Regions.

Local names: Hambeit, Hanbeit, Hamaad, Hammaad, Hammaad el 'eshb.

\section{Ranunculaceae}

*59.65. Nigella sativa $\mathbf{L}$., Sp. Pl., ed. 1, 534 (1753); Boulos 1999, p. 148.

Habitat and life from: Escape from cultivation. Therophyte.

Floristic category: Med and Sah-Sind Regions.

Local name: Habbet el-baraka.

\section{Resedaceae}

60.66. Ochradenus baccatus Delile, Deser. Egypt, Hist. Nat. 63 (1814); Boulos 1999, p. 231.

Habitat and life form: Sandy plains and wadi beds. Nano phanerophyte.
Floristic category: Sah - Sind and IR - Tur Regions.

Local names: Qordi, Garthy, Thanabaan.

61.67. Reseda pruinosa Delile, Descr. Egypte, Hist. Nat. 63 (1814); Boulos 1999, p. 236.

Habitat and life form: Sandy and gravelly deserts and plains. Therophyte.

Floristic category: Sah - Sind Region.

Local names: Denaaba, Danabaan, Denebaan,

Denabaan, Thanabaan, Khosaama.

\section{Scrophulariaceae}

62.68. Kickxia aegyptiaca (L.) Nabelek, Publ. Fac. Sci. Univ. Masaryk Brno 80: 30 (1926) subsp. aegyptiaca; Boulos 2002, p. 65.

Habitat and life form: Desert wadis and sandy plains. Chamaephyte.

Floristic category: Sah-Sind Region.

Local names: Thab el-faar, Ibswaai, 'Eshb eddeeb, Reesha, Doreisha, Megeineena.

63.69. Scrophularia xanthoglossa Boiss., Diagn. Pl. Orient., ser. 1, 12: 38 (1853); Boulos 2002, p. 72.

Habitat and life form: Desert wadis. Chamaephyte.

Floristic category: Sah-Sind Region.

Local names: Barwek, Qortom, Qartam.

\section{Solanaceae}

*63.70. Lycopersicum esculentum Miller, Gard. Dict. ed. 8 (1768); Hepper 1998, p. 55. Habitat and life form: Cultivated vegetable crop Therophyte.

Floristic category: Cultivated.

Local names: Tamatem, Qoutta.

64.71. Hyoscyamus muticus L., Mant. 1: 45 (1767); Boulos 2002, p. 50.

Habitat and life form: Desert wadis and plains. Chamaephyte.

Floristic category: Med and Sah-Sind Regions.

Local names: Semm el-faar, Tatoora, Shagaret es-sakraan, Sakaraan.

\section{Tamaricaceae}

65.72. Tamarix nilotica (Ehrenb.) Bunge, Tent. Tamaric, 54 (1852); Boulos 2000, p. 127.

Habitat and life form: Wide wadis with sandy silty ground . Nanophanerophyte.

Floristic category: Sah - Sind, Sud - Zamb and IR - Tur Regions.

Local names: Abal, Atl, Athal.

\section{Thymelaeaceae}

66.73. Thymelaea hirsuta (L.) Endl., Gen.

Suppl. 4 (2): 65 (1848); Boulos 2000, p. 115. 
Habitat and life form: Sandy and stony plains. Nanophanerophyte.

Floristic category: Med and Sah-Sind Regions. Local names: Mithnaan, Mitnaan.

25. Umbelliferae

67.74. Deverra tortuosa (Desf.) DC., Prodr. 4: 143 (1830); Boulos 2000, p. 167.

Habitat and life form: Desert wadis, sandy and stony plains. Chamaephyte.

Floristic category: Sah-Sind Region.

Local names: Shabat el-gabal, Qozzaah, Qazzaah, Qazooh, Sekoo, Kerddwy, Zakook, Sakeeh, Qeseekh.

*68.75. Foeniculum vulgare Mill., Gard. Dict., ed. 8, no. 1 (1768); Boulos 2000, p. 174.

Habitat and life form: Roadsides,canal banks, hillsides, escape from cultivation. Chamaephyte.

Floristic category: Med and Sah-Sind Regions, cultivated.

Local name: Shamar.

\section{Zygophyllaceae}

69.76. Fagonia arabica L., Sp. Pl., ed. 1, 386 (1753); Boulos 2000, p. 14.

Habitat and life form: Sandy plains and desert wadis. Chamaephyte.

Floristic category: Sah - Sind Region.

Local names: Gamd, Gamda, Gemda, Shibreeq, Shobrooq, 'Aqool el-ghazaal, Halwa, Helwa elgemal, Haad, Shoakaan, Showeika.

69.77. Fagonia bruguieri DC., Prodr. 1:704 (1824); Boulos 2000, p. 15.

Habitat and life form: Sandy plains and desert wadis. Chamaephyte.

Floristic category: Sah - Sind and IR - Tur Regions.

Local names: Khoshyaat, 'Agool, 'Aqool, 'Attan, 'Attani.

69.78. Fagonia mollis Delile, Descr. Egypte, Hist. Nat. 76, t. 27, f. 2 (1814); Boulos 2000, p.18.

Habitat and life form: Sandy and stony desert wadis and plains. Chamaephyte.

Floristic category: Sah - Sind Region.

Local names: Shoka'a, Halaawa, Halaawi.

70.79. Tribulus terrestris L., Sp. Pl., ed. 1, 387 (1753); Boulos 2000, p. 28.

Habitat and life form: Desert plains, waste ground, weed of cultivation. Therophyte.

Floristic category: Med and Sah-Sind Regions.

Local names: Shiqshiq, Dreiss, Kharshoom enna'ga, Daqn esh-sheikh.

71.80. Zygophyllum coccineum L., Sp. Pl., ed. 1, 386 (1753); Boulos 2000, p. 24.
Habitat and life form: Sandy plains. Chamaephyte.

Floristic category: Sah - Sind, IR - Tur and Sud - Zamb Regions.

Local names: Ratrayt, Rotreyt, Balbal, Belbel, Bawaal, Bizz el-Kalba, Hamd.

71.81. Zygophyllum decumbens Delile, Descr. Egypte, Hist. Nat. 221,t.27, f. 3 (1814); Boulos 2000, p.24.

Habitat and life form: Stony wadis and desert plains. Chamaephyte.

Floristic category: Sah - Sind, and Sud - Zamb Regions.

Local names: Khreysy, Khreiza, Khoreize, Khriyet.

71.82. Zygophyllum simplex L., Mant. 68 (1767), Boulos 2000, p. 23

Habitat and life form: Sandy soils. Therophyte. Floristic category: Sah - Sind and IR - Tur Regions.

Local names: Garmal, Qarmal, Goormeil.

\section{DISCUSSION}

The present study on the plant diversity in Wadi Hagul comprises 82 species belonging to 71 genera from one Gymnospermae family and 25 Angiospermae families: Compositae is represented by 20 species, Leguminosae 9 species, Zygophyllaceae 7 species, both of Chenopodiaceae and Gramineae are represented by 6 species. Asclepiadaceae and Polygonaceae are represented by 4 species each. Three species from Cruciferae are recorded. Each of Boraginaceae, Resedaceae, Scrophulariaceae, Solanaceae and Umbelliferae are represented by 2 species; while one species is recorded from each of Capparaceae, Cucurbitaceae, Ephedraceae, Euphorbiaceae, Geraniaceae, Labiatae, Malvaceae, Neuradaceae, Nitrariaceae, Plantaginaceae, Ranunculaceae, Tamaricaceae and Thymelaeaceae. The annual species in the studied area are 33 species; while the perennial ones are 28 species. Nineteen species are recorded as shrubs while the trees are 2 species. Four species recorded from the studied area are considered as a new distribution in the Desert Phytogeographical Region according to Tackholm 1974 and Boulos 2009, which are: Medicago lupulina, Nigella sativa, Lycopersicum esculentum and Foeniculum vulgare.

\section{REFERENCES}

Abdallah M. S., Sa'ad F. M. and Abbas A. S. (1972). Taxonomical studies in the Flora 
of Egypt II-Natural Flora of Egyptian Nubia before the Construction of Aswan High Dam. The Herbarium Tech. Bull. No. 4, 1-83. Dokki, Giza, Egypt.

Ali M. M., Badri M. A., Hassan L. M. and Springuel I. V. (1997). Effect of physiogeographical factors on desert vegetation, Wadi Allaqi Biosphere Reserve, Egypt: a multivariate analysis. Ecologie. 28: 119-128.

Amer W. M. (2004). Floristic Study of ElQantara area, North Sinai with a Special Reference to the Current Human Impacts. Proceedings of First International Conference on Strategy of Egyptian Herbaria March 9-11, pp.191- 212.

Belal A.E. and Springuel, I. (1996): Economic value of plant diversity in arid environments. Nature. and Resources, 32: 33-39.

Boulos L. (1999). Flora of Egypt. vol. I. AlHadara Publishing. Cairo, Egypt.

Boulos L. (2000). Flora of Egypt. vol. 2. AlHadara Publishing. Cairo, Egypt.
Boulos L. (2002). Flora of Egypt. vol. 3. AlHadara Publishing. Cairo, Egypt.

Boulos L. (2005).Flora of Egypt. vol. 4. AlHadara Publishing. Cairo, Egypt.

Boulos L. (2009). Flora of Egypt Checklist. AlHadara Publishing. Cairo, Egypt.

El-Hadidi M. N. (1993). Natural Vegetation, In G. M. Craig (ed.)., The Agriculture in Egypt, Chapter 3: 39-62. Oxford University Press.

Hassan L. M. (1987). Studies on the flora of the Eastern Desert, Egypt. Ph. D. Thesis, Faculty of Science; Cairo University, Egypt.

Mosallam H. A. M. (2007). Assessment of target species in Saint Katherne Protectorate, Sinai, Egypt. Journal of Appl. Sci. Res. June: 456-469.

Soliman M. A. M. (2007). Taxonomical survey of some wadies in South Sinai. Egyp. Jour. of Appl. Sci. 22: 44-63.

Täckholm V. (1974). Students' Flora of Egypt. Second edition, Published by Cairo University, Printed by Cooperative Printing Company, Beirut, Lebanon.

\footnotetext{
دراسة على التنوع النباتى بوادى حجول_الصحراء الشرقيةــ مصر

عبد الحليم عبد المجلى محمد

- ق قسم بحوث الفلورة وتصنيف النباتات معهد بحوث البساتين ـ مركز البحوث الزر اعية ـ الدقىـ الجيزة ـ مصر
}

ملخص

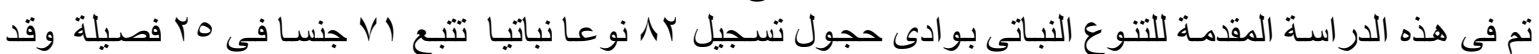

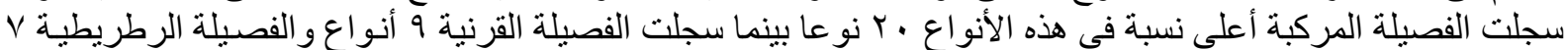

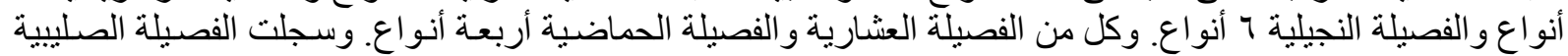

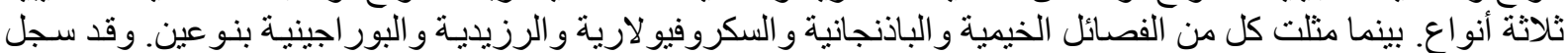

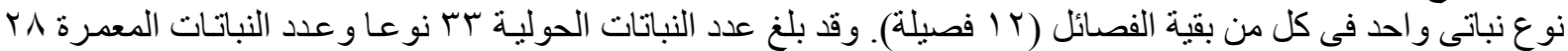

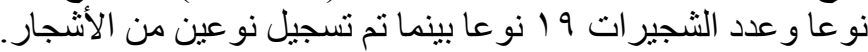

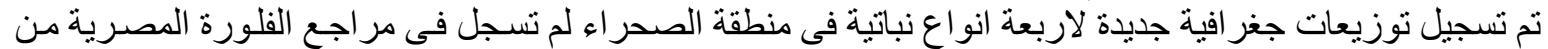

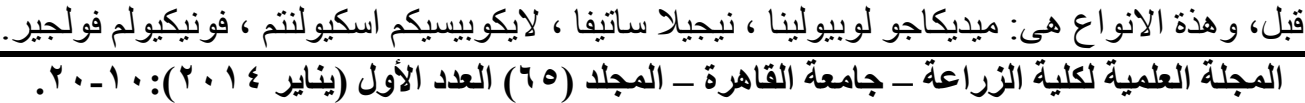

